\title{
Determinants of Advanced Stage at Initial Diagnosis of Breast Cancer in Pakistan: Adverse Tumor Biology vs Delay in Diagnosis
}

\author{
Samina Khokher ${ }^{1 *}$, Muhammad Usman Qureshi $^{2}$, Saqib Mahmood $^{3}$, Sadia Sadiq $^{4}$
}

\begin{abstract}
Background: Breast cancer is the most frequent cancer of women in Pakistan with the majority presenting with stage III or IV lesionsat initial diagnosis. Patient and health system related factors are well known determinants of delay in presentation and diagnosis. Additionally, breast cancer being a heterogeneous disease, the various molecular subtypes featuring different aggressiveness also need to be considered. The present study evaluated the association of stage at initial diagnosis of breast cancer with these two factors in local women at a tertiary level health care facility in Lahore, Pakistan. Materials and Methods: Patient and tumor features were recorded separately during diagnostic workup in Breast Clinics at INMOL and at Services Hospital, Lahore. Data were entered in MS Excel and analyzed by descriptive statistics and Chi-Square test. Results: Among the 261 patients, $64 \%$ were staged as late breast cancer ( $\mathrm{LBC}$ ), the mean age was 46.8 with standard deviation of 13 years. Some $92 \%$ had invasive ductal carcinoma (IDC), $61 \%$ had luminal types (LT) of non-aggressive tumor while 39\% had the non-luminal types (NLT) of of HER2-enriched or basal aggressive tumors. While 70\% of patients presented within one year of symptomatic disease (early report group "ERG"), 30\% reported after a mean delay of 4 years with a standard deviation of 3.75 years. The stage distribution among ERG patients was not statistically different from those reporting late $(P=0.123)$. Statistically larger proportion of patients with NLT presented as $L B C$ as compared to the $L T(P=\mathbf{0 . 0 3 4})$. Among the $E R G$, statistically different stage distribution of disease was observed for the NLT versus LT ( $P=0.047)$. Among those presenting late, this difference was insignificant $(P=0.416)$. Conclusions: Breast cancer is a distinct disease in Pakistan with a high frequency of aggressive molecular types affecting younger women, with the majority presenting as LBC. Association of NLT with higher stage at diagnosis is statistically significant whereas time delay in diagnosis is not. Further research is required to define the risk profile and features in local patients. The burden of $\mathrm{LBC}$ can be reduced by promoting breast health awareness and by establishing easily accessible dedicated breast care set ups in the hospitals.
\end{abstract}

Keywords: Breast cancer - Pakistan - delayed diagnosis - mortality - advanced breast cancer - molecular types

Asian Pac J Cancer Prev, 17 (2), 759-765

\section{Introduction}

Breast cancer is the most prevalent cancer of women worldwide (Siegel et al., 2015). According to the world cancer report developing countries with limited resources have lower incidence but higher mortality rates for this cancer (Boyle and Levin 2008). The same phenomenon has been reported from countries in Asia (Ghoncheh et al 2015). It is the most frequently occurring and the most frequent cause of cancer related deaths of women in Pakistan (Bhurgri et al., 2006; Asif et al., 2014). Advanced stage at initial diagnosis of breast cancer is considered to be the most important factor linked to less favorable outcomes and higher mortality rates for this disease (Bradley et al. 2001). About 40 to $60 \%$ of patients with breast cancer in Pakistan have been reported to be diagnosed when disease has already advanced to Stage III or IV (Gilani et al., 2003; Malik et al., 2010; Khokher et al., 2012). Various causes of this delayed presentation have been described. Lack of screening facilities and lack of infrastructure for early diagnosis is considered to be an important factor for the delay in diagnosis. Lower level of education and lack of awareness, wrong beliefs and initial treatment with traditional medicine, lower socioeconomic status and poor access to health care facility are other factors linked to the delay in diagnosis of the symptomatic disease in most of these developing countries like Pakistan (Igene 2008; Akhtar et al., 2011; Talpur et al., 2011; Khan et al., 2015). All these factors contribute to the time lag between appearance of symptoms in the patients and the time cancer is diagnosed and staged in the health care facility.

${ }^{1}$ Surgical Special Department, Services Hospital, ${ }^{2}$ Nauman Associates, Consulting Acturies, ${ }^{4}$ Surgical Division, INMOL hospital, Lahore, ${ }^{3}$ Department of Human Genetics \& Molecular Biology, University of Health Sciences, Khayaban-e-Jamia Punjab, Pakistan *For correspondence: drsamkhokher@yahoo.com 
With the increasing knowledge of molecular biology, breast cancer is now known to be a heterogeneous disease with many geographic, racial and ethnic variations (Parkin 2004; Carey et al., 2006; Carvalho et al., 2014; Singh et al., 2014; Newman 2015). Perou et al (2000) have described various molecular types on Gene Expression profiling (GEP). Four molecular classes with increasing features of aggressive behavior are known to be; Luminal A, Luminal B, HER2-enriched and Basal or Triple Negative (TN) types of breast cancer (Cianfrocca \& Gradishar 2009; Yersel \& Brutca 2014). Luminal A and B are known to be slow growing and metastasizing late when compared to the HER2- enriched and Basal types of breast cancers. The Luminal types if left without treatment are therefore likely to take longer time to advance into Stage III or IV as compared to the HER2-enriched or Basal/TN types. It is therefore implied that Luminal types of breast cancer have positive association with diagnosis at earlier stages as compared to the Non Luminal types of breast cancer.

Study Aim

The factor of time delay in reporting to health care facility for diagnosis and treatment is an established risk for late stage disease presentation but association with inherent adversity of the tumor type with late stage diagnosis has not been studied well. This study was designed to evaluate the role of the inherent adversity and aggressiveness of the immuno-histochemically defined molecular types of breast cancer for advanced stage disease presentation in comparison with the time delay in diagnosis, in local patients reporting to tertiary care health facilities at Lahore, Pakistan.

\section{Materials and Methods}

Design, setting and ethical consideration: This was an observational, cross sectional study conducted at Breast Clinic in Surgical Division of Institute of Nuclear Medicine and Oncology Lahore (INMOL), from 1st September 2012 to 31st August 2013 and Breast Clinic in Surgical Special Unit at Services Hospital Lahore (SHL) from 1st October 2013 to 31st March 2015. The patients included in the study had their diagnostic workup and treatment as per clinical practice guidelines of the hospital and no additional intervention was done except for data collection for which informed consent was taken. The study was approved by the ethical committee of University of Health Sciences, Lahore.

Diagnostic protocol and data collection: The medical record number, contact number, name, age, menopausal status, family history, the presenting symptom and duration of the presenting symptom of patients were documented in predesigned printed proforma for all female patients attending the breast clinic and clinically suspected to have breast cancer. Informed consent was taken. Triple assessment of patients was done. Core Needle Biopsy was done in patients having tumors with intact skin and incisional biopsy was done in patients having tumors with ulcerated skin. Patients with tumors less than $2 \mathrm{~cm}$ in size had ultrasound guided biopsy. Biopsy specimens, fixed in formalin were sent to the Pathologist for histopathology and immunohistochemistry of ER, PR and HER2 receptors. X-ray of chest, abdominal ultrasound and Bone Scan were done for the metastatic work up. The patients were followed up and reports were entered in the proforma. The male patients and patients lost to the follow up with incomplete diagnostic or metastatic work up were excluded from the study.

Operational Definitions: Family history was categorized as positive when one or more first degree and/or second degree relative suffered from breast cancer and/or ovarian cancer. Clinical Staging of disease was done according to the Tumor (T), Node $(\mathrm{N})$ and Metastasis (M) status described in AJCC version 6 (Singletary \& Connolly 2006). Clinical Stage I and II were grouped as Early Breast Cancer (EBC) while Stage III and IV were grouped as Late Breast Cancer (LBC). The patients were divided into two groups depending upon the duration of presenting symptom as those presenting up to one year as Early Report Group (ERG) and those presenting after one year of the first notice of the symptom as Late Report Group (LRG). ER and PR were considered "Positive" if nuclear staining of $1 \%$ or more cells was positive. Hercep Score 3+ was taken as "Positve", 2+ as "Equivocal", and 0 or $1+$ as "Negative" for HER2. The patients with Equivocal HER2 status on IHC had further FISH analysis where ever possible and definitive HER2 status assigned. The patients were divided into five molecular subclasses according to St Gallen Consensus: Luminal A (ER and/or PR+, HER2 Negative and Grade 1or 2), Luminal B HER2 Negative (ER and/or PR+, HER2 Negative and Grade3), Luminal B HER2 Positive (ER and/or PR+, HER2 positive and any Tumor Grade), HER2 enriched (ER and PR Negative, HER2 positive and any Tumor Grade) and Triple Negative/Basal (ER and PR Negative, HER2 Negative and any Tumor Grade) (Goldhirsch et al 2011). The luminal tumors with ER and/or PR+ but unknown or Equivocal HER2 and/or unknown Grade were grouped as Luminal nonspecific while tumors with ER and PR Negative status and Equivocal or unknown HER2 were grouped as HER2/Basal nonspecific. The four Luminal types (Luminal A, Luminal B HER2 Negative, Luminal B HER2 Positive and Luminal nonspecific) were labeled as Luminal Type (LT) and the three non Luminal types (HER2 enriched, Triple Negative/Basal and HER2/Basal nonspecific) were labeled as Non Luminal Type (NLT).

Statistical Analysis: The data of completed proformas was entered in Microsoft Excel and analyzed by descriptive statistics. Chi square test was applied for the association studies between the molecular subtype and clinical stage at diagnosis of breast cancer as well as for the studies between the time delay in diagnosis and the clinical stage at diagnosis of breast cancer. P-value of less than 0.05 was considered significant.

\section{Results}

The data of 261 patients with complete diagnostic and metastatic work up was available. This included 111 patients from the Breast Clinic at INMOL from 1st September 2012 to 31st August 2013 and 150 patients from the Breast Clinic at SHL, from 1st October 2013 to 31st March 2015 respectively). The patient and tumor 
Determinants of Advanced Stage at Initial Diagnosis of Breast Cancer in Pakistan: Tumor Biology vs Diagnosis Delay Table 1. The Patient and Tumor Features of the Study Group (N=261)

\begin{tabular}{|c|c|c|c|c|c|c|c|c|c|}
\hline \multirow{3}{*}{ Parameter } & \multirow{3}{*}{ Category } & \multicolumn{4}{|c|}{ Luminal } & \multirow{2}{*}{\multicolumn{3}{|c|}{$\begin{array}{c}\text { Basal or HER2-enriched } \\
\text { (Aggressive Tumor Types) }\end{array}$}} & \multirow{3}{*}{ Total } \\
\hline & & \multicolumn{4}{|c|}{ (Non-Aggressive Tumor Types) } & & & & \\
\hline & & A & B HER 2- & B HER 2+ & $\begin{array}{l}\text { Non- } \\
\text { specific }\end{array}$ & Basal & $\begin{array}{l}\text { HER2 } \\
\text { enriched }\end{array}$ & $\begin{array}{l}\text { Non- } \\
\text { specific }\end{array}$ & \\
\hline \multicolumn{2}{|l|}{ Total Frequency } & 54 & 54 & 18 & 34 & 50 & 32 & 19 & 261 \\
\hline \multirow{7}{*}{ Age } & $20-29$ & 1 & 5 & 2 & 4 & 3 & 1 & 2 & 18 \\
\hline & $30-39$ & 10 & 12 & 6 & 3 & 18 & 8 & 4 & 61 \\
\hline & $40-49$ & 17 & 14 & 4 & 10 & 13 & 11 & 5 & 74 \\
\hline & $50-59$ & 15 & 4 & 2 & 11 & 6 & 8 & 6 & 52 \\
\hline & $60-69$ & 6 & 12 & 1 & 4 & 9 & 4 & 2 & 38 \\
\hline & $70-79$ & 4 & 6 & 2 & 2 & 1 & & & 15 \\
\hline & $80-89$ & 1 & 1 & 1 & & & & & 3 \\
\hline \multirow{2}{*}{ Family History } & Positive & 12 & 11 & 5 & 3 & 5 & 6 & 5 & 47 \\
\hline & Negative & 42 & 43 & 13 & 31 & 45 & 26 & 14 & 214 \\
\hline \multirow{3}{*}{$\begin{array}{l}\text { Menopausal } \\
\text { Status }\end{array}$} & Pre & 23 & 30 & 12 & 18 & 28 & 16 & 11 & 138 \\
\hline & Post & 27 & 24 & 5 & 14 & 17 & 12 & 7 & 106 \\
\hline & Hysterectomized & 4 & & 1 & 2 & 5 & 4 & 1 & 17 \\
\hline \multirow{5}{*}{ Histology } & IDC & 42 & 51 & 18 & 31 & 48 & 32 & 19 & 241 \\
\hline & ILC & 10 & 1 & & 1 & & & & 12 \\
\hline & Metaplastic & & 2 & & & 1 & & & 3 \\
\hline & Mix IDC\&ILC & & & & 1 & & & & 1 \\
\hline & Mucinous & 2 & & & 1 & 1 & & & 4 \\
\hline \multirow{4}{*}{ Grade } & 1 & 4 & & & 1 & & & & 5 \\
\hline & 2 & 50 & & 10 & 18 & 10 & 11 & 8 & 107 \\
\hline & 3 & & 54 & 8 & 13 & 39 & 21 & 11 & 146 \\
\hline & Unknown & & & & 2 & 1 & & & 3 \\
\hline \multirow{2}{*}{ ER } & Positive & 54 & 49 & 18 & 34 & & & & 155 \\
\hline & Negative & & 5 & & & 50 & 32 & 19 & 106 \\
\hline \multirow{2}{*}{ PR } & Positive & 49 & 38 & 13 & 32 & & & & 132 \\
\hline & Negative & 5 & 16 & 5 & 2 & 50 & 32 & 19 & 129 \\
\hline \multirow{4}{*}{ HER2 } & Positive & & & 18 & & & 32 & & 50 \\
\hline & Negative & 54 & 54 & & 2 & 50 & & & 160 \\
\hline & Equivocal & & & & 27 & & & 15 & 42 \\
\hline & Unknown & & & & 5 & & & 4 & 9 \\
\hline \multirow{8}{*}{ Stage } & Stage IA & 4 & 1 & 1 & & & & & 6 \\
\hline & Stage IB & 1 & & & & & & & 1 \\
\hline & Stage IIA & 6 & 5 & 2 & 5 & 9 & & 3 & 30 \\
\hline & Stage IIB & 10 & 19 & 3 & 8 & 5 & 8 & 3 & 56 \\
\hline & Stage IIIA & 14 & 11 & 3 & 7 & 12 & 5 & 7 & 59 \\
\hline & Stage IIIB & 8 & 12 & 8 & 8 & 13 & 17 & 3 & 69 \\
\hline & Stage IIIC & 2 & 1 & & 2 & 2 & & 1 & 8 \\
\hline & Stage IV & 9 & 5 & 1 & 4 & 9 & 2 & 2 & 32 \\
\hline \multirow{2}{*}{$\begin{array}{l}\text { Delay in } \\
\text { Reporting }\end{array}$} & Within One Year & 32 & 45 & 15 & 21 & 36 & 24 & 11 & 184 \\
\hline & More than One Year & 22 & 9 & 3 & 13 & 14 & 8 & 8 & 77 \\
\hline
\end{tabular}

Table 2. Association of Stage at Diagnosis with Time Delay Versus Molecular Subtype

\begin{tabular}{|l|c|c|c|c|c|}
\hline \multicolumn{2}{|c|}{} & Stage I - II & Stage III - IV & Total & P-values \\
\hline \multirow{3}{*}{ Luminal Category } & Luminal & 65 & 95 & 160 & \multirow{2}{*}{0.034} \\
\cline { 2 - 6 } & Basal/HER2 & 28 & 73 & 101 & \\
\cline { 2 - 5 } & Total & 93 & 168 & 261 & \\
\hline \multirow{3}{*}{ Reporting Delay } & Within One Year & 71 & 113 & 184 & 0.123 \\
\cline { 2 - 5 } & More than One Year & 22 & 55 & 261 & \\
\cline { 2 - 6 } & Total & 93 & 168 & & \\
\hline
\end{tabular}

features of these 261 patients are shown in Table 1. The age range of these patients was from 22 to 85 and mean age was 46.8 years with standard deviation of 13 years. The most frequent age band for patients was 40 to 49 and nearly half of the patients (53\%) were premenopausal. The tumor type in $61.3 \%$ patients was Luminal/ LT while $38.7 \%$ had Non Luminal tumors (HER2-enriched or Basal type). Family history was positive in $18 \%$ (47/261) and $34 \%$ of these had NLT of tumors as compared to about $40 \%(85 / 214)$ of the patients with Negative family history for breast cancer. Only 3\% patients had Stage I disease while $33 \%, 52 \%$ and $12 \%$ had Stage II, III and IV disease, respectively. About $92 \%$ patients had Invasive Ductal Carcinoma (IDC) while $4.5 \%$ had Invasive Lobular Carcinoma (ILC), $1.5 \%$ had mucinous and the remaining had other histologic types of breast carcinoma.

Among younger patients ( $<40$ years in age), $54 \%$ had LT while $46 \%$ had some tumor type in the NLT. Among the older age patients (age $>40$ years) in this study $64 \%$ had Luminal/LT while $36 \%$ had NLT. 70\% of the patients 
Table 3. Association of Stage at Diagnosis with Molecular Subtype in the Early Versus Late Report Groups

\begin{tabular}{|c|c|c|c|c|c|}
\hline & & $\begin{array}{c}\text { Stage } \\
\text { I-II }\end{array}$ & $\begin{array}{c}\text { Stage } \\
\text { III - IV }\end{array}$ & Total & $\begin{array}{c}\text { P- } \\
\text { values }\end{array}$ \\
\hline \multirow{3}{*}{$\begin{array}{l}\text { Within } \\
\text { One Year }\end{array}$} & Luminal & 50 & 63 & 113 & \multirow{3}{*}{0.047} \\
\hline & $\begin{array}{l}\text { Basal/ } \\
\text { HER2 }\end{array}$ & 21 & 50 & 71 & \\
\hline & Total & 71 & 113 & 184 & \\
\hline \multirow{3}{*}{$\begin{array}{l}\text { More } \\
\text { than One } \\
\text { year }\end{array}$} & Luminal & 15 & 32 & 47 & \multirow{3}{*}{0.416} \\
\hline & $\begin{array}{l}\text { Basal/ } \\
\text { HER2 }\end{array}$ & 7 & 23 & 30 & \\
\hline & Total & 22 & 55 & 77 & \\
\hline
\end{tabular}

reported within one year of the appearance of the first symptom and 39\% of these were found to be at staged I or II after the work up. Among the $30 \%$ of the study population presenting after one year of the appearance of the first symptom named as Late Report Group (LRG), only $29 \%$ had Stage I or II disease. The delay in reporting after appearance of the first symptom, to the tertiary care health facility ranged from more than 1 year to 22 years in the LRG and their mean delay time was 4 years with standard deviation of 3.75 years. The patient, who reported 22 years after noticing a lump in her breast, was an 80 years old lady. She had Luminal A type of breast cancer with Mucinous G I histology and at diagnosis was at T4N2M0 (Stage IIIB).

Table 2 shows the association of the Stage at diagnosis with the NLT of tumors versus LT of breast cancer $(\mathrm{P}=0.034)$ and also with the time delay in reporting the symptomatic disease among ERG versus $\mathrm{LRG}(\mathrm{P}=0.123)$. The subset analysis of ERG versus LRG to evaluate association of Stage at diagnosis with the molecular type of breast cancer among these two groups is shown in Table 3. The association of molecular type with stage at diagnosis was statistically significant $(\mathrm{P}=0.047)$ in the ERG while it was not so in the LRG $(\mathrm{P}=0.416)$.

\section{Discussion}

Stage at diagnosis at the time of treatment is considered to be the single most important factor related to favorable outcomes and longer survival in breast cancer (Bradley et al. 2001). Based upon this concept, screening programs have been established in the developed world. They are practiced to detect early breast cancer at a presymptomatic stage when treatment is associated with the best chances of cure. However, due to lack of screening programs as well as delayed reporting of symptomatic disease in the developing countries, up to $80 \%$ of breast cancer patients are diagnosed at advanced stages (Agarwal et al., 2007; Igene 2008; Echeverria et al., 2009; Moore et al., 2009; Bhikoo et al., 2011). This is in contrast to about $10 \%$ of the patients presenting as advanced breast cancer at initial diagnosis in the developed countries like USA and Sweden (Sariego 2009; Leong et al., 2010). The widely used TNM staging system is an anatomically based classification system which records the loco-regional extent and distant spread of the disease (Singletary \& Connolly 2006). Advanced breast cancer therefore is a clinical entity which includes a wide range of presentations of locally advanced (Stage III) or metastatic (Stage IV) breast cancer. Breast cancer however is a heterogeneous disease consisting of many molecular subtypes which have different risk factors, growth rate, clinical course, metastatic behavior and response to treatment. Therefore in some patients breast cancer may be a biologically indolent, slow growing tumor presenting at advanced stage, years after its first appearance because of delay in reporting to health care facility for diagnosis or in other patients it may be a tumor with rapid growth rate due to intrinsic biologically aggressive nature, growing or spreading rapidly and presenting at late stage of disease in a short span of time.

The delay in reporting to appropriate health care facility is known to be an established important determinant of late diagnosis at advanced stages in the limited resource countries (Igene 2008; Akhtar et al., 2011; Talpur et al., 2011; Khan et al., 2015). In the present study, an 80 years old patient gave history of lump in her breast for the last 22 years, which was diagnosed as T4N2M0, tumor of Luminal A variety. It may sound incomprehensible that a woman can delay seeking medical advice for such a long period of time in the developed societies but many similar cases with neglected huge ulcerated masses in the breast have been reported in literature from the low income, limited resource developing countries (Ishikawa et al., 2002; Khokher et al., 2010; Bhikoo et al., 2011). Various factors have been studied as contributing factors for delayed reporting of symptomatic breast cancer in the developing countries. Some of them are patient related and some are health system related and many are socioeconomic or cultural in nature. Patient related factors include lower level of education and lack of awareness, wrong beliefs, initial treatment with traditional medicine, lower socio-economic status etc (Gorin et al., 2006; Aziz et al., 2008; Khokher et al., 2011; Khan et al 2015). Health system related factors include lack of screening facilities to diagnose asymptomatic disease and lack of dedicated breast clinics or diagnostic set ups with facilities for breast imaging, where triple assessment can be performed to diagnose symptomatic disease. All these factors contribute in one way or the other to time delay in presentation to health care facility for definitive diagnosis and treatment.

Our study supports time delay to be an important but not statistically significant determinant of diagnosis at advanced stages of breast cancer in local population. Earlier report of symptomatic breast cancer logically has the potential to be diagnosed at early stage. In the present study, ERG made up $70 \%$ of the patients but majority of them $(61 \%)$ presented at stage III or IV. Among the LRG (30\% of the study patients) $71 \%$ presented at stage III or IV. Although the chances of higher stage disease differ in ERG and LRG (61\% and 71\%, respectively) however the difference is not statistically significant $(\mathrm{P}=0.123)$.

When data of ERG and LRG was analyzed separately, a statistically significant majority of patients: $70 \%$ (50/71) with aggressive molecular subtypes in the NLT were diagnosed at Stage III or IV in the ERG as compared to the 56\% (63/113) of the non aggressive Luminal subtypes. Among the LRG, 77\% (23/30) of the aggressive molecular 
types were diagnosed as LBC as compared to the $68 \%$ $(32 / 47)$ of the non aggressive subtypes. It is therefore inferred that when there is prolonged delay ( $>1$ year) in the diagnosis of symptomatic breast cancer, higher frequency of patients having aggressive as well as non aggressive molecular subtypes are likely to be diagnosed as LBC and the different biological course of two molecular types loses statistical difference on stage at diagnosis. However if the symptomatic disease is diagnosed earlier on $(<1$ year) there are significantly more chances of nonaggressive Luminal molecular types to be diagnosed at earlier stages of I or II.

Based upon Gene Expression Profiling (GEP) various molecular types have been identified (Perou et al., 2000) and this has made a paradigm shift in the current breast cancer research. GEP is expensive and not available in the clinical setting even in the developed countries and therefore surrogate molecular classes of breast cancer have been described for clinical use (Goldhirsch et al., 2011). The breast cancers are classifiable in these groups clinically if we have information about the histologic grade $(\mathrm{G})$ of tumor as well as the three receptors (Estrogen receptor (ER), Progesterone receptor (PR) and Human Epidermal Growth factor Receptor 2 (HER2) status on immunohistochemistry.

Racial and ethnic disparities in breast cancer have been reported in studies upon multi racial and multi ethnic societies in the developed countries. NLT are more frequent among the younger patients and among the African Americans and Asian populations living in these countries (Merkin 2002; Parkin 2004; Carey et al., 2006; Carvalho et al., 2014; Singh et al., 2014; Newman 2015). The same phenomenon has been reported in patients with breast cancer in Pakistan and some Asian countries (Khokher et al., 2010; Kakarala et al., 2010). As these tumors grow and spread rapidly within a short span of time, higher frequency of these tumors is likely to be an important determinant of late stage disease presentation in these populations. The higher frequency of these molecular types among the patients of breast cancer in the developing countries has been reported however association of LBC presentation with NLT is not well studied in these countries. The patients of our study group had higher proportion of NLT (39\%) as compared to patients in the developed countries and majority of our patients (64\%) were diagnosed as LBC. In addition, our study group of breast cancer patients differs from those in the developed world by virtue of being younger with higher frequency of IDC. These cancers have different risk profile and biological features (Li et al., 2006; Phipps et al., 2011). The patients having NLT in our study were more likely to be diagnosed as LBC at Stage III or IV $(\mathrm{P}=0.034)$ in comparison with the LT. Our study therefore supports the higher frequency of occurrence of NLT in local patients. Moreover association of NLT with late stage diagnosis in local population is statistically significant $(\mathrm{P}=0.034)$.

The delay in diagnosis of breast cancer in the developing countries is generally portrayed as the most important determinant of LBC presentation. However our study shows it to be a factor of lesser importance as compared to the intrinsic aggressiveness of the molecular subtypes of breast cancer in local population. In our study the patients who reported symptomatic disease within one year were not statistically different from those who delayed reporting for more than one year, with regard to the Stage distribution of disease $(\mathrm{P}=0.123)$. Moreover within the ERG there was statistically different stage distribution of disease for the NLT versus LT $(\mathrm{P}=0.047)$ as compared to the LRG where the difference was insignificant $(\mathrm{P}=0.416)$.

The burden of higher stage disease of LBC is associated with disease related suffering, higher cost of care and mortality which needs to be addressed in Pakistan. Diagnosing and treating Stage I disease is the most cost effective breast cancer intervention (Groot et al., 2006). The gold standard strategy to downstage this disease is establishment of screening program to diagnose asymptomatic disease. However, it is difficult to make huge investments for this in a developing country like Pakistan with limited resources (Badar et al., 2011) and therefore earlier diagnosis of symptomatic disease is recommended as a promising and cost-effective alternative approach. The higher frequency of NLT and disease affecting younger population necessitates diagnosis and appropriate treatment in shortest possible time in this population. This can be achieved with increasing awareness and education level of women and establishment of dedicated well equipped breast clinics in all the public sector hospitals where trained staff can provide rapid diagnostic workup and state of the art care for these patients. Research is required to identify the causes and risk factors of development of aggressive molecular subtypes in young women of this country to plan effective preventive strategy.

Our study conducted over a period of two and a half years has the limitation of small number of patients but has the strength of homogeneous population and single team providing diagnostic workup with follow up treatment in these patients in tertiary level health care facilities at Lahore, Pakistan. The present study classified the breast tumors into molecular subtypes clinically using information about the histologic grade $(G)$ of tumor as well as the immune-histochemistry of three receptors (Estrogen receptor (ER), Progesterone receptor (PR) and Human Epidermal Growth factor Receptor 2 (HER2) rather than the Gene Expression Profiling (GEP) described for the molecular classification of breast cancers (Perou et al., 2000). GEP has definitely made a paradigm shift in the current breast cancer research but it is expensive and not yet available in the clinical setting even in the developed countries and therefore surrogate molecular classes of breast cancer have been described for clinical use (Goldhirsch et al., 2011). In the present study, ERG included patients reporting to tertiary health care facility within one year and LRG included those reporting more than one year after appearance of any disease symptom. It can be argued that even one year is too long a delay to report symptomatic disease, however this was an arbitrary cut off and further studies are required for patients reporting within one year of appearance of symptomatic 
disease. Another limitation of our study was that $19.5 \%$ (51) of the study patients could not be categorized specifically because of equivocal (42) or unknown (9) HER2 status. Therefore based upon their ER and PR status, these tumors were classified as Luminal nonspecific or Basal/HER2 nonspecific. Cost issues for FISH testing were the key limiting factor. It is not unusual to manage patients of breast cancer without HER2 in routine clinical setting in the developing countries where expensive tests and drugs cannot be afforded by a significant number of patients (Aziz 2008).

In conclusions, Breast Cancer is a biologically distinct disease in Pakistan. Here it affects younger women and higher proportion of these patients is diagnosed to have IDC and Non Luminal aggressive types of tumor. Majority of patients present with advanced stage III or IV disease.

Time delay in diagnosis due to various reasons is known to be as an established determinant of diagnosis at late stages. However, our study shows statistically insignificant association of time delay with stage at diagnosis as compared to the statistically significant association of aggressive Non Luminal types of tumors with higher stage at diagnosis of breast cancer.

Larger studies are required to confirm these findings as well as research to define the risk profile and features of breast cancer in local population so that an effective preventive strategy can be adopted. Higher frequency of NLT of breast cancers, progressing rapidly and presenting as advanced stage disease, necessitates setting up of well equipped dedicated breast clinics in all public sector hospitals, so as to expedite the diagnosis of disease and facilitate appropriate treatment.

\section{Acknowledgements}

Authors acknowledge the Directors of INMOL; Dr Saeeda Asghar and Dr Abubaker Shahid for establishing and facilitating Breast Clinic at INMOl and the Principal of SIMS, Professor Hamid Mahmood Butt for facilitation of Breast Clinic at SHL. Authors wish to thank the nurses; Nusrat Alam and Naheed Kanwal at INMOL \& Irum and Gulshan at SHL for their help in record keeping in the respective Breast Clinics as well as their efforts for the patient follow up. Authors declare no competing interest and there were no grants for the study.

\section{References}

Agarwal G, Pradeep PV, Aggarwal V, Yip CH, Cheung PS (2007). Spectrum of breast cancer in Asian women. World J Surg, 31, 1031-40.

Akhtar M, Akulwar V, Chandak K, Gandhi D (2011). Is locally advanced breast cancer a neglected disease? Indian J Cancer, 48, 403-5.

Asif HM, Sultana S, Akhtar N, Rehman JU, Rehman RU (2014). Prevalence, risk factors and disease knowledge of breast cancer in Pakistan. Asian Pac J Cancer Prev, 15, 4411-6

Aziz Z (2008). Across generations: cancer treatment in developing countries. J Clin Oncol, 26, 4990-1.

Aziz Z, Iqbal J, Akram M (2008). Effect of social class disparities on disease stage, quality of treatment and survival outcomes in breast cancer patients from developing countries. Breast, 14, 372-5.

Badar F, Faruqui ZS, Uddin N, Trevan EA (2011). Management of breast lesions by breast physicians in a heavily populated south asian developing country. Asian Pac J Cancer Prev, 12, 827-32.

Bhikoo R, Srinivasa S, Yu T Moss D, Hill AG (2011). Systematic review of breast cancer biology in developing countries (Part 2): Asian subcontinent and South East Asia. Cancer, 3, 2382-401.

Bhurgri Y, Bhurgri A, Nishter S, et al (2006). Pakistan - country profile of cancer and cancer control 1995-2004. J Pak Med Assoc, 56, 124-30.

Boyle P, Levin B. World Cancer Report (2008). Lyon, france: international agency for research on cancer; 2008.

Bradley CJ, Given CW, Roberts C. (2001) Disparities in cancer diagnosis and survival. Cancer, 91, 178-88.

Carey LA, Perou CM, Livasy CA, et al (2006). Race, breast cancer subtypes and survival in the carolina breast cancer study. JAMA, 295, 2492-502.

Carvalho FM, Bacchi LM, Pincerato KM, Rijn MVD, Bacchi CE (2014). Geographic differences in the distribution of molecular subtypes of breast cancer in Brazil. BMC Women's Health, 14, 102.

Cianfrocca MO, and Gradishar W (2009). New molecular classifications of breast cancer. CA Cancer J Clin, 59, 303-13.

Echeverria SE, Borrell LN, Brown D, Rhodes G (2009). A local area analysis of racial, ethnic and neighbourhood disparities in breast cancer staging. Cancer Epidemiol Biomarkers Prev, 18, 3024-9.

Ghoncheh M, Hafshejani AM,Salehiniya H (2015). Incidence and mortality of breast cancer and their relationship to development in Asia. Asian Pac J Cancer Prev, 16, 6081-7.

Gilani GM, Kamal S, Akhter AS (2003). A differential study of breast cancer patients in Punjab, Pakistan J Pak Med Assoc, 53, 478-80.

Goldhirsch A, Wood WC, Coates AS, et al (2011). Strategies for subtypes-dealing with the diversity of breast cancer: highlights of the St Gallen International expert consensus on the primary therapy of early breast cancer 2011. Ann Oncol, 22, 1736-47.

Gorin SS, Heck JE, Cheng B, Smith SJ (2006). Delays in breast cancer diagnosis and treatment by racial/ethnic group. Arch Intern Med, 166, 2244-52.

Groot MT, Baltussen R, Groot CAU, Anderson BO, Hortobagyi GN (2006). Costs and health effects of breast cancer interventions in epidemiologically different regions of Africa, North America and Asia. Breast, 12, 81-90.

Igene $H$ (2008). Global health inequalities and breast cancer: an impending public health problem for developing countries. Breast, 14, 428-34.

Ishikawa T, Hamaguchi Y, Ichikawa Y, et al (2002). Locally advanced mucinous carcinoma of the breast with sudden growth acceleration: a case report. Jpn J Clin Oncol, 32, 64-7.

Kakarala M, Rozek L, Cote M, Liyanage S, Brenner DE (2010). Breast cancer histology and receptor status characterization in Asian Indian and Pakistani women in the U.S. a SEER analysis. BMC Cancer, 10, 191.

Khan TM, Leong JPY, Ming LC, Khan AH (2015). Association of knowledge and cultural perception of Malaysian women with delay in diagnosis and treatment of breast cancer: a systematic review. Asian Pac J Cancer Prev, 16, 5349-57.

Khokher S, Mahmood S, Khan SA (2010). Response to Neoadjuvant chemotherapy in patients with advanced breast cancer: A local hospital experience. Asian Pac J Cancer 

Prev, 11, 303-8.

Khokher S, Qureshi W, Mahmood S, Saleem A, Mahmud S (2011). Knowledge attitude and preventive practices of women towards breast cancer in educational institutions of Lahore, Pakistan. Asian Pac J Cancer Prev, 12, 2419-24.

Khokher S, Qureshi MU, Riaz M, Akhtar N, Saleem A (2012). Clinicopathologic profile of breast cancer patients in Pakistan: Ten years data of a local cancer hospital. Asian Pac J Cancer Prev, 13, 693-8

Leong SPL, Shen ZZ, Liu TJ, Agarwal G, et al (2010). Is breast cancer the same disease in Asian and Western countries? World J Surg, 34, 2308-24.

Li CI, Daling JR, Malone KE, Bernstein L, et al (2006). Relationship between established breast cancer risk factors and risk of seven different histologic types of invasive breast cancer. Cancer Epidemiol Biomarkers Prev, 15, 946-54.

Malik AM, Pathan R, Shaikh NA, Qureshi JN, Talpur KA (2010). Pattern of presentation and management of Ca Breast in developing countries. There is a lot to do. J Pak Med Assoc, 60, 718-721.

Merkin SS, Stevenson L, Powe N (2002). Geographic socioeconomic status, race and advanced stage breast cancer in New York city. Am J Pub Health, 92, 64-70.

Moore MA, AriyaratneY, Badar F, et al (2009). Cancer epidemiology in South Asia- Past, present and future. Asian Pac J Cancer Prev, 10, 49-67.

Newman LA (2015) Disparities in breast cancer and African ancestory: Global perspective. Breast, 21, 133-9.

Parkin DM (2004). International variation. Oncogene, 23, 6329-40.

Perou CM, Sorlie T, Eison MB, et al (2000). Molecular portraits of human breast tumors. Nature, 406, 747-52

Phipps AI, Buist DS, Malone KE, et al (2011). Reproductive history and risk of three breast cancer subtypes defined by three biomarkers. Cancer Causes Control, 22, 399-405.

Sariego J (2009). Pattern of breast cancer in United States: Does geography matter? Am Surg, 75, 545-50.

Siegel RL, Miller KD, Jemal A (2015). Cancer statistics 2015. CA Cancer J Clin, 65, 5-29.

Singh M, Ding Y, Zhang LY, et al (2014). Distinct breast cancer subtypes in women with early-onset disease across races. Am J Cancer Res, 4, 337-52.

Singletary SE, Connolly JL (2006). Breast cancer staging: working with the sixth edition of the AJCC cancer staging manual. CA Cancer J Clin, 56, 37-47.

Talpur AA, Srahio AR, Ansari A, Ghumro AA (2011). Late Presentation of breast cancer: a Dilemma.JPMA, 61, 662-6.

Yersal O and Barutca S (2014). Biological subtypes of breast cancer: Prognostic and therapeutic implications. World $J$ Clin Oncol, 5, 412-24. 